\title{
Vorwort
}

\section{ZIC̈ gif}

\section{Gabriel M. Ahlfeldt • Konstantin A. Kholodilin • Wolfgang Maennig}

Immobilienökonomik, also die Lehre über die gesellschaftlichen Entscheidungen darüber, weshalb in welchem Umfang Immobilien wie, wann, wo und zu welchem Zweck produziert und bewirtschaftet werden, und welche Wirkungen davon auf andere volkswirtschaftliche Bereiche ausgehen, wurde an den deutschen Universitäten bis Anfang der 1990er Jahre nur wenig beachtet. Seitdem ist zwar einiges geschehen, aber gemessen an der Bedeutung der Immobilienwirtschaft, beispielsweise gemessen am Anteil an der Wertschöpfung oder eben auch an den Anteilen, welche die Haushalte aus ihrem verfügbaren Einkommen für das Wohnen ausgeben, ist der Umfang an Forschung und Lehre an den deutschen Universitäten und Forschungsinstituten weiterhin gering.

Dennoch gibt es auch in Deutschland und im Ausland mittlerweile eine Reihe ausgewiesener deutschstämmiger Experten, welche eine immobilienwirtschaftliche Forschung betreiben, die auch internationalen akademischen Standards genügt. Ziel dieses Sonderhefts ist es solchen Experten Gehör zu verschaffen, die robuste Forschungsergebnisse auf internationalem Niveau vorgelegt haben. Solche Experten wurden von uns eingeladen, zu ausgewählten Themen der Wohnungspolitik den internationalen Stand der Forschung zusammenzufassen und relevante Politikimplikationen allgemeinverständlich herauszuarbeiten. Dadurch soll der Wissenstransfer

G. M. Ahlfeldt $(\square)$

Department of Geography and Environment, London School of Economics (LSE), Houghton

Street, London, WC2A 2AE, Großbritannien

E-Mail: g.ahlfeldt@lse.ac.uk

K. A. Kholodilin

DIW Berlin, Mohrenstraße 58, 10117 Berlin, Deutschland

E-Mail: kkholodilin@diw.de

W. Maennig

Department of Economics, Universität Hamburg, Von-Melle-Park 5, 20146 Hamburg, Deutschland

E-Mail: wolfgang.maennig@uni-hamburg.de 
aus der akademischen Forschung in die deutsche Wohnungspolitik erleichtert und die öffentliche Debatte um eine wichtige Stimme bereichert werden.

Die Zeit ist günstig. Denn auch in der deutschen Öffentlichkeit ist die volkswirtschaftliche Betrachtung der Immobilien inzwischen hochaktuell. Einige große Städte sind in den letzten Jahren im wahrsten Sinne des Wortes ,attraktiv“ geworden; sie zeichnen sich durch den Zuzug von Menschen aus dem In- und/oder Ausland aus. Niedrige Zinsen und die internationale Wettbewerbsposition Deutschlands haben dazu geführt, dass selbst Menschen und Institutionen, die selber keine deutschen Immobilien nutzen wollen, diese investiv interessant finden.

Diese Attraktivität hat, wie Matthias Wrede in diesem Band erinnert, ihren Preis: so kam es in vielen deutschen Städten zu steigenden Mieten und Immobilienpreisen. Wrede verdeutlicht die Interdependenzen zwischen Arbeits- und Immobilienmärkten in verschiedenen Regionen auf und zeigt, dass die Änderung in einem Markt Reaktionen auf anderen Märkten auslöst. Wird eine Anpassung der Marktpreise beispielsweise durch eine Mietpreisbremse verhindert, verschwindet die gesteigerte Attraktivität nicht. Stattdessen ergeben sich beispielsweise längere Suchzeiten auf den Märkten für Mietwohnungen, oder die Suchenden sind gezwungen, in die Eigentumsmärkte auszuweichen oder in andere, eventuell umliegende Regionen zu pendeln. Eine Umverteilung gibt es also auch bei Preisregulierungen - es werden immobile Mieter zumindest kurzfristig gewinnen, mobile Outsider verdrängt. Die Wohnraumknappheit wird jedoch nicht verringert, sondern erhöht.

Diese, auf Interdependenzen zielenden theoretischen Aspekte werden von Lorenz Thomschke ergänzt um eine Diskussion von empirischen Studien zu Mietpreisregulierungen. Die deutsche Mietpreisbremse von 2015 hat nach den meisten Studien die Mietentwicklung gegenüber den ,ungebremsten“ Mietwohnungen um rund $3 \%$ gedämpft. Dies gilt zumindest in Städten mit einem qualifizierten Mietspiegel und insbesondere im oberen Preissegment, weniger jedoch in den unteren Preissegmenten, die für viele einkommensschwächere Haushalte relevant sind. Und selbst der beobachtete mietpreisdämpfende Effekt bedeutet nicht, dass Haushalte mittleren und niedrigen Einkommens mit Wohnraum versorgt werden. Letzteren wird durch die Preisregulierung die Möglichkeit genommen, sich im Wettbewerb um eine Wunschwohnung gegen einkommensstärkere und bildungsnähere Mitbewerber durchzusetzen, selbst wenn sie eine höhere Zahlungsbereitschaft besitzen. Wird der Preismechanismus ausgeschaltet, verlagern sich die Auswalkriterien der Vermieter weg von den finanziellen Kriterien in Richtung sozialer Status. Thomschke hält die angedachte Ausweitung der Geltungsdauer für ,ein gewagtes Unterfangen“, solange keine taugliche Datenbasis für eine Evaluierung der Mietpreisbremse vorhanden ist.

Christian Hilber setzt einen Schritt vorher an, indem er anhand empirischer Studien aufzeigt, dass Angebotsbeschränkungen in Form von Bodennutzungsregulierungen und geografischen Barrieren zur Erschließung und Bebauung von Flächen in Boomphasen ursächlich für (stärker) steigende Immobilienpreise sein können. Er zeigt - Achtung, Anhänger von starken Regulierungen der Baupolitik! -, dass ökonomische Konjunkturzyklen in stark regulierten Metropolregionen zu ausgeprägteren Preiszyklen führen. Und langfristig steigen Immobilienpreise in Städten mit begrenztem Angebot stärker. Hilber zeigt aber auch, dass es darüber hinaus ,,endo- 
gen getriebene“ Zyklen gibt, die wenig mit solchen „realen“ Restriktionen zu tun haben.

Die weltweite Finanz- und Wirtschaftskrise von 2008 und in den Folgejahren wird teilweise als Beispiel solcher endogen getriebener „Preisblasen“ gesehen, und Konstantin A. Kholodilin und Claus Michelsen schätzen mit Daten für 20 OECDLänder, dass beispielsweise eine um einen Prozentpunkt höhere Wachstumsrate des Bruttoinlandsprodukts die Wahrscheinlichkeit einer gleichzeitig auftretenden Spekulationsblase um gut sechs Prozentpunkte steigert. Wird die Kreditvergabe in Relation zum Bruttoinlandsprodukt verdoppelt, wächst die Blasenwahrscheinlichkeit im Fall von Hypothekarkrediten um 47,5 Prozentpunkte. Wichtig im Zusammenhang mit der Blasenbildung ist auch, dass sich die Wahrscheinlichkeit einer spekulativen Preisblase um ca. $37 \%$ erhöht, wenn die Vorperiode durch die Blasenentwicklungen gekennzeichnet war. Sie zeigen sodann, dass nunmehr zumindest in acht Ländern erneut die Gefahr einer Blasenbildung herrscht. Die Entwicklung in Deutschland halten sie im Vergleich zu anderen Ländern aufgrund der relativ soliden Finanzierungsverhältnisse für nicht allzu bedenklich. Sie sehen jedoch gewisse Gefahren in den Metropolen. Sie empfehlen, in Deutschland das makroprudenzielle Instrumentarium der Kreditvergabe zu schärfen und zu präzisieren, wann die BaFin ihre Befugnisse nach $\S 48 \mathrm{u}$ KWG, den Fremdkapitalanteil bei Immobilienfinanzierungen zu begrenzen, ausüben darf.

Im Zusammenhang mit der Preis- und insbesondere der Blasenbildung sind zwei Faktoren bedenkenswert, die bislang nicht im Zentrum der Aufmerksamkeit stehen. Zum einen ist die Schaffung von möglichst viel Transparenz bedeutsam, auch mithilfe einer ausgewogenen Medienberichterstattung. Stephan Heblich zeigt am Beispiel des Fracking - der Erschließung von Schiefergas - in England, dass dort Ängste vor möglichen Risiken, geschürt durch die Medien, zu überschießenden Immobilienpreisreaktionen führen konnten. Ein geringfügiges Erdbeben der Stärke 1,7-2,5, welches für die Substanz der betroffenen Immobilien unbedenklich ist, führte im Verbreitungsgebiet lokaler Zeitungen $\mathrm{zu}$ - nicht nur vorübergehenden - Preisverringerungen von 3-5\%. An diesem Beispiel lässt sich lernen, dass mit Transparenz die Unsicherheit verringert werden kann. Dazu gehören neben einer laufenden Kommunikation der Entwicklungen auf den Immobilienmärkten bei jeglichen größeren technischen oder Infrastrukturmaßnahmen plausible Darstellungen der zu erwartenden Kosten und Risiken, aber eben auch der Chancen und ökonomischen Nutzen.

Zum anderen ist im Zusammenhang mit Blasenbildungen bedeutsam, dass in Deutschland eher pessimistische Erwartungen bezüglich zukünftiger Immobilienpreisentwicklungen herrschen. Dies zeigen Niklas Gohl, Peter Haan, Claus Michelsen und Felix Weinhardt anhand einer Analyse mit Daten aus einer repräsentativen Befragung des SOEP. Sie zeigen auch, dass Befragte ausländischer Herkunft in Deutschland optimistischere Preiserwartungen haben. Neben der Wirkung solcher Erwartungen auf die Wahrscheinlichkeit von Blasenbildungen sind diese Erwartungen bedeutsam für die Erklärung der relativ geringen Quote von selbstgenutztem Wohneigentum. Bisherige Erklärungsansätze der geringen deutschen Eigennutzerquoten, welche Variablen wie das Einkommen, die Altersstruktur der Bevölkerung, das Kreditvergabeverhalten der Banken, das Steuersystem oder regulatorische Ein- 
griffe oder Transfers zugunsten von Mietern heranziehen, bedürfen also einer Ergänzung.

Die international vergleichbar geringe Eigennutzerquote in Deutschland von rund $50 \%$ ist erstaunlich vor dem Hintergrund, dass rund $84 \%$ der Deutschen eine eigene Wohnung bevorzugen. Aus der Politik kommen offensichtlich zu wenig Hilfen für die Bürger, ihre Wünsche zu erfüllen. In Städten wie Berlin, wo die Eigennutzerquote mit unter $20 \%$ besonders gering ist, versuchen Politik und Verwaltung einen Anstieg gar mit Umwandlungsverboten etc. zu verhindern. Gabriel M. Ahlfeldt und Wolfgang Maennig zeigen auf, dass zu den üblichen Argumenten pro höherer Eigennutzerquoten (z. B. Zustand der Immobilien und soziales Engagement der Bewohner) ein weiteres Argument zu beachten ist: Geringe Eigennutzerquoten können die Stadtentwicklung behindern, die in der Regel zur Verbesserung der Lebenssituation beitragen soll. Solche Verbesserungen gehen mit Miet- und Preissteigerungen einher. Wenn die gestiegene lokale Attraktivität eines „Kiezes“ neue Mieter mit besonders hohen Präferenzen für die entwickelte Gegend anzieht, wird die Miete in der Regel sogar stärker steigen als der Lagewertigkeits-Effekt aus Sicht der Alt-Mieter. Eine Opposition der Mieter gegen Aufwertung der eigenen Nachbarschaft wird dadurch rational, aber eine verbesserte Eigenheim-Politik könnte das Problem lindern. Ahlfeldt und Maennig zeigen mögliche Politikmaßnahmen auf und plädieren an diejenigen Parteien, welche weniger eigennutzer-freundlich sind, zu überprüfen, ob sie damit die Ziele ihrer Mitglieder und Wähler erreichen.

Preisentwicklungen (und die teilweise daraus abgeleiteten Preiserwartungen) spielen in den zuvor beschriebenen Entscheidungen der Individuen eine wichtige Rolle. Auch wenn sich bei den hierzu veröffentlichten Daten in den letzten Jahren einiges getan hat, ist der Fortschritt zum Beispiel bei den Bodenwertermittlungen in Deutschland bislang relativ gering. In Zukunft könnten in Deutschland verstärkt standardisierte, über statistische Schätzmodelle getriebene Bodenwertermittlungen betrieben werden, wie dies in einer Reihe von anderen Ländern wie beispielsweise den USA, Australien, Kanada, Neuseeland, Dänemark, Finnland, Nordirland bereits der Fall ist. Gegenüber der bisherigen Methode anhand von Ermittlungen über Gutachterausschüsse hätte dies den Vorteil, dass die Modelle und ihre Spezifikationen transparent nachvollziehbar sind und weniger von der Qualität der lokalen Gutachterausschüsse abhängt. Jens Kolbe, Rainer Schulz, Martin Wersing und Axel Werwatz zeigen anhand von Schätzungen für Berlin, dass solche statistischen Massenbewertungssysteme in der Lage sind, Bodenwertschätzungen zu erstellen, die den bisherigen Einschätzungen des Berliner Gutachterausschusses qualitativ nicht nachstehen. Unter Umständen kommt hier mit der Reform der Grundsteuer, die ab 2025 umgesetzt werden muss und bei der die aktuellen (Boden)Werte eine größere Rolle spielen, verstärkt Änderungsdruck in Richtung mehr Konsistenz und Transparenz auf. An den Kosten dürfte es nicht scheitern: Die Kosten pro Objekt dürften bei etwa $20 €$ pro Bewertung liegen, was einem Prozent der durchschnittlichen Steuereinnahmen von $2000 €$ pro Immobilie entspricht.

In Deutschland wurde bislang vor allem die Kausalitätsrichtung von der allgemeinen Wirtschaft auf die Immobilienwirtschaft diskutiert. Erst seit der Krise ab 2008 wird deutlich, dass die Kausalität auch von der Immobilienwirtschaft in Richtung allgemeine Wirtschaft laufen kann, und dass Immobilien und deren Preisentwicklung 
über die Versorgung der Bevölkerung mit Wohn- und Arbeitsmöglichkeiten hinausgehende Wirkungen haben. Drei solcher Aspekte werden im vorliegenden Band diskutiert. Erstens zeigt Johannes Stroebel, dass es neben der bislang diskutierte Kausalitätsrichtung von der allgemeinen Inflation auf die Immobilienpreise (z. B. „optimaler Inflationsschutz“) auch die andere Wirkungsrichtung gibt: Das mit steigenden Immobilienpreisen erhöhte Vermögen führt zu erhöhter Konsumnachfrage. Die Einzelhändler können höhere Aufschläge auf ihre Einkaufspreise („markups“) durchsetzen, was die Preise für Konsumgüter erhöht. Die Inflation steigt, und die realen Wirkungen einer expansiven Geldpolitik werden geschwächt. Einen Verteilungseffekt gibt es auch zu berücksichtigen: In Regionen, in denen die Immobilienpreise steigen, kann sich dies bei hinreichend hohen Eigennutzerraten negativ auf das Realeinkommen der Mieter auswirken - selbst dann, wenn Mietpreisbremsen wirken sollten. Diesem Nebeneffekt steigender Immobilienpreise ist bisher wenig Aufmerksamkeit geschenkt worden.

Einen zweiten Aspekt beleuchtet Rainald Borck, der empirische Evidenz vorlegt, wonach größere und dichter besiedelte Städte zwar absolut mehr Luftverschmutzung und $\mathrm{CO}_{2}$ und teilweise höhere Schadstoffkonzentration (siehe die deutsche Dieseldiskussion) verursachen, aber weniger $\mathrm{CO}_{2}$ pro Kopf. Er zeigt - in weiten Teilen Deutschlands könnte dies zu Erstaunen führen -, dass Städte zu wenig dicht bebaut werden und im Vergleich zum Wohlfahrtsoptimum zu klein sind, wenn nicht durch eine ökonomisch begründete Stadtplanung eingegriffen wird. Solche lokalen Verdichtungen werden von der OECD und anderen Organisationen im Rahmen von „compact city“-Initiativen gefordert. Die von der OECD angestoßenen Diskussionen um die Akademisierung der Bildungspolitik zeigt, dass es zwar dauern wird, bis dies in Deutschland ankommt - verhindern lässt es sich aber kaum.

Drittens gehen Franz Fuerst und Ben Dalton auf den Aspekt von Immobilien und Nachhaltigkeit ein. Die - gemessen an den $\mathrm{CO}_{2}$-Reduktionszielen der Politik - zu geringe Rate von ökologischen Sanierungen in Deutschland deutet darauf hin, dass aus Sicht der Entscheider solche Investitionen nicht angemessen honoriert werden. Dies erstaunt zunächst, da Fuerst und Dalton in einer Meta-Studie, welche 42 wissenschaftliche Veröffentlichungen auswertet, zu dem Ergebnis kommen, dass die Mieten in energieeffizienten Immobilien um rund 6\% höher liegen - im Wohnbereich sogar um über $8 \%$. Die Verkaufspreise von energieeffizienten Immobilen liegen um rund 7,6\% höher, im Wohnbereich um 5,5\%. Auch für deutsche Märkte liegen entsprechende Studien vor, wenngleich implizite Zahlungsbereitschaft von Mietern unter dem Niveau der potentiellen einsparbaren Energiekosten liegt. Allerdings nehmen die ,grünen“ Prämien mit der Zeit ab, und die Prämien beinhalten meistens auch Nachhaltigkeitsfaktoren wie Wasserverbrauch, Luftqualität und Baumaterialien.

Es bleibt zu hoffen, dass durch dieses Sonderheft der Zeitschrift für Immobilienökonomie die Diskussion um volkswirtschaftliche Themen der Immobilien angeregt und an dem einen oder anderen Punkt versachlicht wird. Hinreichende Kompetenz deutscher Wissenschaftler, dies soll der Band ebenfalls verdeutlichen, ist jedenfalls an in- und ausländischen Universitäten und Forschungsinstituten vorhanden.

Open Access Dieser Artikel wird unter der Creative Commons Namensnennung 4.0 International Lizenz (http://creativecommons.org/licenses/by/4.0/deed.de) veröffentlicht, welche die Nutzung, Vervielfäl- 
tigung, Bearbeitung, Verbreitung und Wiedergabe in jeglichem Medium und Format erlaubt, sofern Sie den/die ursprünglichen Autor(en) und die Quelle ordnungsgemäß nennen, einen Link zur Creative Commons Lizenz beifügen und angeben, ob Änderungen vorgenommen wurden. 